\title{
Large Lepton Number and High Temperature Symmetry Breaking in MSSM
}

\author{
Borut Bajc日 \\ J. Stefan Institute, 1001 Ljubljana, Slovenia \\ Goran Senjanoviç \\ International Center for Theoretical Physics, 34100 Trieste, Italy
}

\begin{abstract}
It is known that a large neutrino number, of the order of a few percent of the entropy of the universe, leads to symmetry breaking at high temperature. We show here that in the minimal supersymmetric standard model (MSSM) this implies the breaking of electromagnetic charge invariance at $T \gg M_{W}$ allowing for the solution of the monopole problem.
\end{abstract}

\footnotetext{
${ }^{1}$ borut.bajc@ijs.si

${ }^{2}$ goran@ictp.trieste.it
} 


\section{Introduction}

Although we know the baryon number of the universe with a fair precision, $B / S \approx 10^{-9 \pm 1}$ ( $S$ is the entropy of the universe), remarkably enough we know almost nothing about the lepton number. The only thing we can say with some confidence is the observational limit [1, 2, 3, 4]

$$
L \lesssim S
$$

The determination of $L$ remains one of the main challenges for cosmology. Of course, it is just a part of what is probably the main missing link for the standard big-bang model of cosmology: the detection of the predicted neutrino sea. It is worth commenting that from the electric charge neutrality of the universe we know that the electron number is as small as the proton number. The lepton number of the universe, if not small, must be in the form of neutrinos.

Now, one often runs into an almost textbook argument (erroneous) that $|L| \approx|B|$, due to the anomaly of $B+L$. Namely, the $B+L$ violating processes at $T \gg M_{W}$ are presumably in equilibrium, so that $B+L$ gets washed out: $B+L \approx 0$. This is wrong, since the cosmology of $L$ large is rather subtle. It is known that in the standard model a large $L \gtrsim S / 100$ leads to non-restoration of the $\mathrm{SU}(2)_{L}$ gauge symmetry at $T \gg M_{W}$ [5, [0], and thus the sphaleron processes remain exponentially suppressed [7], much as at $T=0$. More precisely, if $L \lesssim S / 100$, then it is true that $B+L$ will be washed out and $L / S \approx 10^{-10}$, but in the opposite case one has a completely consistent cosmological scenario.

Thus, we should be open minded and allow for a possibility of $L / S$ not being small. The question, of course, is how natural is to have a substantial $L / S$. Well, in the context of supersymmetric theories, there is a simple mechanism of Affleck and Dine [8] of producing large $L$ and $B$ through flat directions [9]. The challenge, really, is to understand how come $B$ is so small. This important issue remains out of the scope of our work; instead, we just pursue the possibility that $L$ is large.

We are motivated, in large part, by a desire to solve the infamous monopole problem of grand unified theories. Monopoles get over produced during

the phase transition in the early universe when one goes from the unbroken to the broken phase. If the symmetries are not restored at high $T$ [10, 11, then 
the non-restoration of GUTs will solve the monopole problem [12]. Another alternative possibility is that $\mathrm{U}(1)_{e m}$ is broken above $T \approx M_{W}[13]$, so that the monopoles can be annihilated through the cosmic strings which interpolate between monopoles and anti-monopoles. Although it appears difficult to non-restore gauge symmetries in ordinary theories [14], in supersymmetry this may happen quite naturally through the presence of flat directions [15, 16]. The learned reader may know of the no-go theorem for symmetry non-restoration in supersymmetry [17, 18. Although there has been some controversy in this issue [19], it is believed that without flat directions internal symmetries in SUSY are always restored [20].

On the other hand a large lepton number, as we mentioned before, provides a natural way of non-restoring symmetries. This has been recently addressed in the context of the minimal standard model, where one concludes that the Higgs sector must be enlarged, and unfortunately in an arbitrary fashion [6]. Since the phenomenon of symmetry non-restoration with an external charge works as well in supersymmetry [21, it is natural to see what happens in the minimal supersymmetric standard model (MSSM). It turns

out that the MSSM is tailor-fit for this task. Both the electromagnetic charge invariance and the $\mathrm{SU}(2)$ weak symmetry are necessarily broken at high $T$ for sufficiently large lepton asymmetry.

Another interesting application of the large lepton number is in the ultrahigh energy cosmic rays, as suggested in [22]. Here the lepton number is used to help understand the cosmic rays with energies in excess of the GreisenZatsepin-Kuzmin cutoff.

\section{$2 \quad$ MSSM at large $L$ and large $T$}

It is well known that the charged scalar field will have a non-vanishing vev at high temperature if the background charge of the universe is large enough. Take a simple example of a global U(1) symmetry with a field $\phi$ carrying a nonvanishing charge. From

$$
V(\phi)=m^{2}|\phi|^{2}+\lambda|\phi|^{4}
$$

one gets an effective potential at high $T(\gg m)$ and a charge density $n$ [23, 24] 


$$
V_{\text {eff }}=-\mu^{2}|\phi|^{2}+\frac{\lambda}{3} T^{2}|\phi|^{2}+\lambda|\phi|^{4}-\mu^{2} \frac{T^{2}}{6}+\mu n,
$$

where $\mu$ is the chemical potential. It is clear that for $\mu>\sqrt{\lambda / 3} T$, the $\mathrm{U}(1)$ symmetry will be necessarily broken even at $T \gg m$.

This simple fact suffices to establish that $\mathrm{U}(1)_{e m}$ would be broken in the MSSM for large enough background lepton number. Namely, both the lefthanded slepton doublet $\tilde{L}$ and the right-handed selectron $\tilde{e}^{c}$ will get vevs; this implies the complete breaking of $\mathrm{SU}(2)_{L} \times \mathrm{U}(1)_{Y}$ gauge invariance.

The interesting property of this scenario is that not just the sleptons, but also the Higgses and the squarks $\tilde{q}$ learn about the lepton number through the gauge interactions and can in principle develop non-vanishing vevs. In order to solve the monopole problem, we need to make sure that for $M_{W} \ll$ $T<M_{X}$, the unbroken group contains no U(1) subgroup.

The question is if the possible breaking of $\mathrm{SU}(3)_{c}$ through $<\tilde{q}>\neq 0$ can leave a $\mathrm{U}(1)$ subgroup, and the answer is no, as we show now. A first $<\tilde{q}>\neq 0$ breaks $\mathrm{SU}(3)_{c}$ to its $\mathrm{SU}(2)$ subgroup (no $\mathrm{U}(1)$ factor remains) and the rest of the squarks are either doublets or singlets under this group. The doublets, if they get vevs, break $\mathrm{SU}(2)$ completely. Depending on who gets the vev (if any), $\mathrm{SU}(3)_{c}$ is either preserved, or broken down to $\mathrm{SU}(2)$, or broken completely.

In short, whatever the original GUT symmetry is, at $T \gg M_{W}$ there is no $U(1)$ factor and the monopoles, if at all created, necessarily annihilate through the cosmic strings attached to monopole-anti-monopole pairs.

Now, we wish to compute the critical lepton number density which suffices to have $\mathrm{U}(1)_{e m}$ broken for $T \gg M_{W}$.

A toy model: leptons only. To understand what is going on and for the sake of illustration we present first the one generation model of leptons only. This is truly a toy model for not only there are no quarks and Higgses, but as such the model is not anomaly free. We ignore this issue here since for us this is only a pedagogical illustration of what happens in the consistent and realistic theory discussed below.

The $T=0$ potential contains only the D-terms

$$
V^{(D)}=\frac{g^{\prime 2}}{2}\left(\left|\tilde{e}^{c}\right|^{2}-\frac{1}{2}|\tilde{L}|^{2}\right)^{2}+\frac{g^{2}}{8}|\tilde{L}|^{4} .
$$


The high temperature correction to the effective potential can be obtained from 25

$$
\Delta V_{T}^{(D)}=\frac{g^{\prime 2} T^{2}}{2}\left(\left|\tilde{e}^{c}\right|^{2}+\frac{1}{4}|\tilde{L}|^{2}\right)+\frac{3 g^{2} T^{2}}{8}|\tilde{L}|^{2} .
$$

Clearly, the above terms $V^{(D)}$ and $\Delta V_{T}^{(D)}$ imply symmetry restoration at high temperature, a well known fact in supersymmetric theories.

Next, we need the corrections due to the nonzero lepton charge. This is usually done by introducing a chemical potential for each conserved charge [6], thus one for the lepton number $\left(\mu_{L}\right)$ and one for the hypercharge $\left(\mu_{Y}\right)$, which gives [23, 24, 26]

$$
\begin{aligned}
\Delta V_{n}= & -\left(-\mu_{L}+\mu_{Y}\right)^{2}\left|\tilde{e}^{c}\right|^{2}-\left(\mu_{L}-\frac{\mu_{Y}}{2}\right)^{2}|\tilde{L}|^{2} \\
& -\left(-\mu_{L}+\mu_{Y}\right)^{2} \frac{T^{2}}{4}-\left(\mu_{L}-\frac{\mu_{Y}}{2}\right)^{2} \frac{T^{2}}{2}+\mu_{L} n_{L} .
\end{aligned}
$$

As one can see, the role of chemical potential is to provide negative mass square for the sleptons, which per se would imply symmetry breaking. Of course, whether or not the symmetry is broken, depends whether or not the chemical potential is bigger than the temperature.

Notice that we did not include the chemical potential for the weak isospin. The reason for this will be clear from what follows below. Also, we neglected the $\mu^{4}$ terms, which arise from the fermion loops; we checked however, that such an approximation is safe and changes the results only by a few percent. For this reason we will not include them in the rest of the paper.

Now it is more convenient to work directly with the lepton density and to eliminate chemical potentials. After all, the latter are derived quantities; the physical quantity is the lepton charge asymmetry. From the constraints [23, 24]

$$
\frac{\partial \Delta V_{n}}{\partial \mu_{i}}=0, i=L, Y
$$

we get

$$
\Delta V_{n}=\frac{2 n_{L}^{2}}{T^{2}+2|\tilde{L}|^{2}}+\frac{n_{L}^{2}}{T^{2}+4\left|\tilde{e}^{c}\right|^{2}}
$$


Notice again that the charge density prefers nonvanishing vevs and the issue now is whether $n_{L}$ is bigger than $\approx T^{3}$ or not. The total effective potential

$$
V_{e f f}=V^{(D)}+\Delta V_{T}^{(D)}+\Delta V_{n}
$$

is minimized when $\partial V_{e f f} / \partial|\phi|=0\left(\phi=\tilde{e}^{c}, \tilde{L}\right)$, i.e. when

$$
\begin{aligned}
& 2\left|\tilde{e}^{c}\right|\left[g^{\prime 2}\left(\left|\tilde{e}^{c}\right|^{2}-\frac{|\tilde{L}|^{2}}{2}\right)+\frac{g^{\prime 2}}{2} T^{2}-\frac{4 n_{L}^{2}}{\left(T^{2}+4\left|\tilde{e}^{c}\right|^{2}\right)^{2}}\right]=0, \\
& 2|\tilde{L}|\left[\frac{g^{2}}{4}|\tilde{L}|^{2}-\frac{g^{\prime 2}}{2}\left(|\tilde{e}|^{2}-\frac{|\tilde{L}|^{2}}{2}\right)+\frac{g^{\prime 2}+3 g^{2}}{8} T^{2}-\frac{4 n_{L}^{2}}{\left(T^{2}+2|\tilde{L}|^{2}\right)^{2}}\right]=0 \text {. }
\end{aligned}
$$

The solution depends on the value of $n_{L}$. At small (compared with $T^{3}$ ) lepton charge density the vevs of $\left|\tilde{e}^{c}\right|$ and $|\tilde{L}|$ are trivially zero. Since $g^{\prime 2}<$ $\left(g^{\prime 2}+3 g^{2}\right) / 4$ (we will use throughout the values $g^{\prime} \approx 0.36, g \approx 0.65$ ) the first vev to become nonzero with growing $n_{L}$ is $\left|\tilde{e}^{c}\right|$. This happens for $n_{L}>n_{L}^{0}$, where

$$
n_{L}^{0}=\frac{g^{\prime}}{2 \sqrt{2}} T^{3} \approx 0.13 T^{3}
$$

is thus the charge density above which the $\mathrm{U}(1)$ symmetry is spontaneously broken. Naively one could think that this is enough to solve the monopole problem. However, as mentioned above, if the $\mathrm{SU}(2)$ symmetry is not broken, the sphalerons become operative and wash out any nonzero $B+L$, destroying our scenario. Thus the $\mathrm{SU}(2)$ symmetry must also be broken through a nonvanishing vev of $|\tilde{L}|$. This happens when the charge density is bigger than the critical value $n_{L}^{c}$ which is obtained by equating to zero the expressions in parenthesis in (10)-(11) for $|\tilde{L}|=0$. The equation for $n_{L}^{c}$ one gets is of the third order, whose analytic solution is not very illuminating. The numerical result is

$$
n_{L}^{c} \approx 0.20 T^{3}
$$

In order to see whether such a value is experimentally allowed, one must calculate the entropy density at $T \gg M_{W}$, when our analysis make sense. Since in the early universe the entropy density $s \approx 100 T^{3}$ for the MSSM, 
it is clear that the critical density (13) is well below the experimental limit $n_{L} \lesssim 100 T^{3}$ from (1).

The reader may now ask why we did not include a chemical potential also for the third component of the weak isospin. The point is, that it is zero as long as the symmetry is conserved, i.e. for all $n_{L}$ up to the critical charge density (13). This is different from the hypercharge chemical potential, entirely due to the non abelian character of the weak isospin. Since our aim was to calculate the critical charge density, we can assume from the beginning a vanishing weak isospin chemical potential.

MSSM: one-generation case. Let us now study in detail the complete case of 1-generation MSSM. Although the effect will be roughly the same as in the previous model, the exact values of the critical charges will turn out to differ up to a factor of 4 . This surprisingly large number is unexpected: a closer inspection shows that it is due to the large number of degrees of freedom (especially in connection with the hypercharge) carried by the rest (mainly quarks superfields) of the MSSM spectrum. The fact confirm our findings in [6], i.e. that all the degrees of freedom must be taken into account, even those, such as quarks, that do not carry lepton number.

In order to simplify the computation we will consider the following special case.

a) The number of symmetries (chemical potentials) are taken as small as possible. Since the disappearance of Yukawa couplings brings new symmetries, we will study the MSSM effective potential for $T \lesssim 10^{7} \mathrm{GeV}$ and for $\tan \beta=1$. In this case all the Yukawa couplings are effective, i.e. their interactions $|y|^{2} T$ are bigger than the Hubble constant $H \approx T^{2} / M_{P l}$. Raising the temperature would make of course some Yukawas drop out of equilibrium, giving new conserved $\mathrm{U}(1)$ symmetries and thus new chemical potentials. Although straightforward, such a computation would not bring more insight into the problem, so we will limit ourselves to low enough temperatures (but of course still larger than $M_{W}$ ).

b) In the MSSM with the vanishing soft terms and zero $\mu$ term in the superpotential there are two extra symmetries, one of which is an R-symmetry. In the above temperature regime neither of these symmetries is conserved: the interactions that violate them have the effective rate $\Gamma \approx \mu^{2} / T$, with $\mu \approx 10^{2} \mathrm{GeV}$. Clearly this is bigger than the expansion rate of the universe. This fact simplifies further the calculations, so that only three U(1) symmetries need to be considered: lepton number, baryon number and hypercharge. 
As in our previous toy model example, chemical potentials connected to non abelian diagonal generators are zero as long as these symmetries are unbroken.

c) Now that we found the relevant symmetries involved, we can forget the small contributions in the superpotential (even the top Yukawa coupling turns out to be irrelevant) and take simply $W=0$.

d) The 1-generation MSSM gives a very good estimate for the critical charge. We thus present our computations only for this case, but give at the end the numerical results for the realistic case of 3 generations.

We have seen in the previous example that in order to determine the charge $n_{L}^{0}$, above which the first field gets a nonzero vev, one needs to consider only the quadratic (in fields) terms in the effective potential.

The first of two different such terms is the leading 1-loop high temperature corrections to the scalar mass term (a special case is (5))

$$
\Delta V_{T}^{(D)}=\frac{1}{2} \sum_{a, i} C_{2}^{a}\left(r_{i}\right) g_{a}^{2} T^{2}\left|\phi_{i}\right|^{2},
$$

where $\phi_{i}$ are the fields in the representation $r_{i}$ of the gauge group, $a$ runs over different gauge groups with coupling constants $g_{a}$ and $C_{2}^{a}\left(r_{i}\right)=\left(Y_{i} / 2\right)^{2}$ for $\mathrm{U}(1)$ and $3 / 4,4 / 3$ for the fundamental representation of $\mathrm{SU}(2), \mathrm{SU}(3)$ respectively. Written explicitly, (14) has the form

$$
\begin{aligned}
\Delta V_{T}^{(D)}=\frac{T^{2}}{2} & {\left[\left(\frac{g^{\prime 2}}{4}+\frac{3 g^{2}}{4}\right)\left(\left|H_{u}\right|^{2}+\left|H_{d}\right|^{2}+|\tilde{L}|^{2}\right)+g^{\prime 2}\left|\tilde{e}^{c}\right|^{2}\right.} \\
& +\left(\frac{g^{\prime 2}}{36}+\frac{3 g^{2}}{4}+\frac{4 g_{s}^{2}}{3}\right)|\tilde{Q}|^{2} \\
& \left.+\left(\frac{4 g^{\prime 2}}{9}+\frac{4 g_{s}^{2}}{3}\right)\left|\tilde{u}^{c}\right|^{2}+\left(\frac{g^{\prime 2}}{9}+\frac{4 g_{s}^{2}}{3}\right)\left|\tilde{d^{c}}\right|^{2}\right] .
\end{aligned}
$$

The other term needed to determine $n_{L}^{0}$ is a generalization of (6). Using the rules developed in [23, 24, 26] one can work out a general expression for the contribution of the chemical potentials to the effective potential 27.

$$
\Delta V_{n}=-\frac{1}{2} \mu_{a} \mathcal{M}_{a b} \mu_{b}+\mu_{a} n_{a}
$$


with

$$
\mathcal{M}_{a b}=\frac{T^{2}}{6}\left(\sum_{i} f_{a}^{i} f_{b}^{i}+2 \sum_{i} b_{a}^{i} b_{b}^{i}\right)+2 \sum_{i} b_{a}^{i} b_{b}^{i}\left|\phi_{i}\right|^{2} .
$$

The indices $a, b$ run over the conserved symmetries $\left(\mu_{a}\right.$ are the corresponding chemical potentials), while $i$ runs over the different fields; $f_{a}^{i}\left(b_{a}^{i}\right)$ is the $a$-th charge of the $i$-th fermion (boson) field; $\phi_{i}$ is the vev of the $i$-th boson field and $n_{a}$ is the value of the total $a$-th charge asymmetry (we will assume that only the lepton charge asymmetry is nonzero). We should stress that the formulae (16) and (17) are valid in any model, supersymmetric or not. Notice however, that we have neglected higher orders in $\mu$. The next term, $\mu^{4}$, will be shown to be much smaller than the terms in (16) in our case.

Using the usual constraints

$$
\frac{\partial \Delta V_{n}}{\partial \mu_{a}}=0 \rightarrow \mu_{a}=\left(\mathcal{M}^{-1}\right)_{a b} n_{b}
$$

(16) becomes

$$
\Delta V_{n}=\frac{1}{2} n_{a}\left(\mathcal{M}^{-1}\right)_{a b} n_{b} .
$$

If there is no R-symmetry, then $f_{a}^{i}=b_{a}^{i}\left(=q_{a}^{i}\right)$ and

$$
\mathcal{M}_{a b}=\frac{T^{2}}{2}\left(\sum_{i} q_{a}^{i} q_{b}^{i}\right)+2 \sum_{i} q_{a}^{i} q_{b}^{i}\left|\phi_{i}\right|^{2} .
$$

The meaning of (20) can be already obtained in the toy model (see (60). In that case the charges are $q_{L}=-1,1, q_{Y}=1,-1 / 2$ for the singlet and the doublet respectively. Of course, in such a simple model it is much more transparent not to use the complicated general formula (20).

The analog of $n_{L}^{0}$ shown in (12) is given by the minimal charge satisfying at least one of the equations

$$
\left.\frac{\partial V_{e f f}}{\partial\left|\phi_{i}\right|^{2}}\right|_{\left|\phi_{j}\right|^{2}=0}=0
$$

where the relevant terms in $V_{\text {eff }}$ are given by (14) and (19). To understand the meaning of this equation it may help to know that in the toy model 
discussed above (21) reduce to the expressions in the square brackets in (10)(11) with vanishing vevs.

If we write (17) or (20) in a compact form

$$
\mathcal{M}_{a b}=\frac{T^{2}}{2} \mathcal{A}_{a b}+\sum_{i} \mathcal{B}_{a b}^{i}\left|\phi_{i}\right|^{2}
$$

and use the relation

$$
\left.\frac{\partial}{\partial\left|\phi_{i}\right|^{2}} \mathcal{M}^{-1}\right|_{\left|\phi_{j}\right|^{2}=0}=-\left(\frac{2}{T^{2}}\right)^{2} \mathcal{A}^{-1} \mathcal{B}^{i} \mathcal{A}^{-1},
$$

we get

$$
n_{L}^{0}=\min _{i}\left[\frac{T^{6}\left(\sum_{a} C_{2}^{a}\left(r_{i}\right) g_{a}^{2}\right)}{4\left(\mathcal{A}^{-1} \mathcal{B}^{i} \mathcal{A}^{-1}\right)_{L L}}\right]^{1 / 2} .
$$

Using the fields' charges of Table 1 it is now straightforward to calculate the matrices $\mathcal{A}$ and $\mathcal{B}$ and find out that $n_{L}^{0}$ is given by the value, when the right-handed selectron (just as in the toy model) begins to get a nonzero vev:

$$
n_{L}^{0}=\sqrt{2} g^{\prime} T^{3} \approx 0.50 T^{3} .
$$

For $n_{L}>n_{L}^{0}, \mathrm{U}(1)_{Y}$ is spontaneously broken by $\left|\tilde{e}^{c}\right|^{2} \neq 0$. Notice that $n_{L}^{0}$ in the 1-generation MSSM (25) is exactly 4 times bigger than the one found in the simplified model with only leptons (12). This may be somewhat of a surprise. After all, quarks and Higgs superfields carry no lepton charge, so why should their presence matter so much? The fact that they do matter was already noticed in our previous work on the standard model [6], but here the effect is even more dramatic. The point is that any particle with non-zero hypercharge learns about the lepton number through its interactions with the photon and the $Z$ boson.

To get the critical charge $n_{L}^{c}$, where also $\mathrm{SU}(2)_{L}$ gets broken presumably by $|\tilde{L}|^{2}$, one must take into account that now $\left|\tilde{e}^{c}\right|^{2} \neq 0$. For this reason the full dependence of $V_{\text {eff }}$ on $\left|\tilde{e}^{c}\right|^{2}$ must be retained for $n_{L}>n_{L}^{0}$, but for $n_{L}<n_{L}^{c}$ only the quadratic $\left|\phi_{i}\right|^{2}$ term needs to be considered for all the remaining scalar fields (as was the case for all the fields when $n_{L}<n_{L}^{0}$ ).

From the general D-term 


\begin{tabular}{|c|c|c|c|c|c|c|c|c|}
\hline $\mathcal{Q}$ & $e^{c}$ & $L$ & $H_{d}$ & $H_{u}$ & $Q$ & $u^{c}$ & $d^{c}$ & name \\
\hline 1 & -1 & 1 & 0 & 0 & 0 & 0 & 0 & $L$ \\
\hline 2 & 0 & 0 & 0 & 0 & $1 / 3$ & $-1 / 3$ & $-1 / 3$ & $B$ \\
\hline 3 & 1 & $-1 / 2$ & $-1 / 2$ & $1 / 2$ & $1 / 6$ & $-2 / 3$ & $1 / 3$ & $Y / 2$ \\
\hline
\end{tabular}

Table 1: The relevant charges $q_{i}^{a}(a=L, B, Y / 2$, while $i$ goes over the chiral superfields) in MSSM.

$$
V^{(D)}=\frac{1}{2} \sum_{a} D^{a} D^{a}, \quad D^{a}=-g_{a} \phi_{i}^{\dagger}\left(T^{a}\right)_{i j} \phi_{j},
$$

with $\left(T^{a}\right)_{i j}$ the generators of the group in the matrix representation (equal to $Y / 2$ for $\mathrm{U}(1)_{Y}$ and $\tau^{a} / 2, \lambda^{a} / 2$ for the fundamental representation of $\mathrm{SU}(2)_{L}$, $\mathrm{SU}(3)_{c}$ respectively) the following D-terms from (26) are relevant for the determination of the critical charge

$$
\frac{g^{\prime 2}}{2}\left|\tilde{e}^{c}\right|^{4}+g^{\prime 2}\left|\tilde{e}^{c}\right|^{2}\left(-\frac{1}{2}|\tilde{L}|^{2}-\frac{1}{2}\left|H_{d}\right|^{2}+\frac{1}{2}\left|H_{u}\right|^{2}+\frac{1}{6}|\tilde{Q}|^{2}-\frac{2}{3}\left|\tilde{u}^{c}\right|^{2}+\frac{1}{3}\left|\tilde{d}^{c}\right|^{2}\right) .
$$

The critical charge is the minimal charge which satisfies at least two equations among (21), one of which is for $i=\left|\tilde{e}^{c}\right|$ and $\left|\tilde{e}^{c}\right|^{2} \neq 0$ now. One has thus two equations for the two unknowns, $\left|\tilde{e}^{c}\right|^{2}$ and $n_{L}^{c}$ : first

$$
\frac{\partial V_{e f f}}{\partial\left|\tilde{e}^{c}\right|^{2}}\left(\left|\tilde{e}^{c}\right|^{2}, n_{L}^{c}\right)=0
$$

and second, the equation among

$$
\frac{\partial V_{e f f}}{\partial\left|\phi_{i}\right|^{2}}\left(\left|\tilde{e}^{c}\right|^{2}, n_{L}^{c}\right)=0
$$

for $i \neq\left|\tilde{e}^{c}\right|$ with the smallest solution for $n_{L}^{c}$. Now, if you see a huge shark in your immediate vicinity while swimming far away from the shore, what do you do? A correct answer to this question gets you a free coffee from either of the authors. After having established that you are following this stuff, let us go on. 
The effective potential is found from (15), (19) and (27)

$$
V_{e f f}=V^{(D)}+\Delta V_{T}^{(D)}+\Delta V_{n}
$$

Using the notation

$$
V_{T}=V^{(D)}+\Delta V_{T}^{(D)}
$$

one can write down the equations (28), (29) more explicitly as

$$
\frac{\partial V_{T}}{\partial\left|\phi_{i}\right|^{2}}\left(\left|\tilde{e}^{c}\right|^{2}\right)=\frac{\left(n_{L}^{c}\right)^{2}}{2}\left[\left(\frac{T^{2}}{2} \mathcal{A}+\mathcal{B}^{\left|\tilde{e}^{c}\right|}\left|\tilde{e}^{c}\right|^{2}\right)^{-1} \mathcal{B}^{i}\left(\frac{T^{2}}{2} \mathcal{A}+\mathcal{B}^{\left|\tilde{e}^{c}\right|}\left|\tilde{e}^{c}\right|^{2}\right)^{-1}\right]_{11}
$$

It is now straightforward to find out numerically that the first equation to be satisfied among (29) is for $i=|\tilde{L}|$, as expected, which breaks the $\mathrm{SU}(2)_{L}$ gauge symmetry. The value of the critical charge comes out to be

$$
n_{L}^{c} \approx 0.58 T^{3}
$$

which is almost 3 times bigger than in the toy model (13). For $n_{L}>n_{L}^{c}$ both $\mathrm{SU}(2)_{L}$ and $\mathrm{U}(1)_{Y}$ are broken, solving thus the monopole problem.

At this point one can also check the numerical value of the $\mu^{4}$ terms neglected in (16). For example from [26] one finds that for each fermion the ratio between the $\mu^{4}$ term and $\mu^{2}$ term is $[\mu /(\pi T)]^{2}$, which turns out to be always smaller than 0.02 for $n_{L} \leq n_{L}^{c}$, which we considered above. This is somewhat different from a similar calculation in the non-supersymmetric standard model [6], where a larger charge, which is needed to break the $\mathrm{SU}(2)$ symmetry, leads to larger chemical potentials and thus the quartic terms turn out to be still important (corrections to the critical charge in SM are sometimes of order $50 \%$ ).

Furthermore, the inclusion of the Yukawa couplings does not change the numerical results for the critical charge: in fact, the value of the lepton Yukawa coupling is far too small to contribute considerably, while the top Yukawa does not enter the relevant equations, since the quarks and the Higgses do not get a nonzero vev at this stage (we have checked explicitly this statement confirming the above results). Obviously, since the Yukawa interaction is unimportant, it is irrelevant which generation is to be considered in this one-generation MSSM. 
The three-generations realistic case. The last generalization to be done is to include all three generations. This we did numerically along the lines described above. We assumed that the lepton charge is stored in only one of the three generations (remember that there are three conserved lepton number symmetries for vanishing or very small neutrino masses). The more complicated case with more than one non-vanishing lepton asymmetry can be treated along the same lines as here, but in our opinion it would teach us nothing new. Furthermore, one expects no significant change in our results (we will see below that going from one to three generations makes very little difference). The shark is still here: what are you doing about it?

The results are given in Table (2) together with the previously obtained critical charges. The relative error between the results from the 1-generation and 3-generations MSSM are of the order of $20 \%$, which is not much, comparable to other errors we can expect (higher loop contributions, etc.). We can thus say, that the much simpler 1-generation MSSM gives a very good estimate of the correct result. This result confirms the approximate value for the critical charge which leads to the breaking of the $\mathrm{SU}(2)_{L}$ symmetry in the non-supersymmetric standard model [6]. Recall, however, that in the non-supersymmetric version the electromagnetic invariance is not broken and thus the monopole problem is not solved. Thus supersymmetry is seen to play an essential role in our considerations. Without it one would have to postulate an ad hoc existence of light charged scalar particles if one wants the electromagnetic invariance spontaneously broken at high temperature.

\begin{tabular}{|l|c|c|c|}
\hline & toy model & 1-gen. MSSM & 3-gen. MSSM \\
\hline$n_{L}^{0} / T^{3}$ & 0.13 & 0.50 & 0.43 \\
\hline$n_{L}^{c} / T^{3}$ & 0.20 & 0.58 & 0.72 \\
\hline
\end{tabular}

Table 2: The charges, above which the $\mathrm{U}(1)\left(n_{L}^{0}\right)$ and $\mathrm{SU}(2)\left(n_{L}^{c}\right)$ symmetries are broken, from the models described in the text. 


\section{Discussion and outlook}

The phenomenon of symmetry breaking at high temperature plays an important role in cosmology. First, it provides a simple way-out of the monopole problem in grand unification. Furthermore, it changes completely the conventional scenario of baryogenesis, since it implies a freezing of sphaleron effects at all temperatures. A particularly simple realization of this mechanism is provided by the large lepton number of the universe. In this paper we have shown how a sufficiently large lepton number implies the complete breaking of $\mathrm{SU}(2) \times \mathrm{U}(1)$ symmetry in the MSSM for $T \gg M_{W}$. This is achieved through nonzero VEVs of at least one left-handed and one righthanded sleptons.

The critical value for the lepton density of the universe is in complete agreement with the observation. We hope that our work provides a further impetus for the improvement of the existing experimental limits. On the other hand, we need a more fundamental explanation for the large lepton number and the small baryon number of the universe. We hope that this is only a matter of time, or, that we will learn that the lepton number of the universe is small too. It should be mentioned, though, that even a small lepton number would not necessarily imply that our scenario is completely wrong. In fact, in order to have the $\mathrm{SU}(2)_{L} \times \mathrm{U}(1)_{Y}$ symmetry completely broken, one does not need to have necessarily a large lepton number: what is important is to have a large conserved charge density, whatever it is [28]. For example, a large $R$ charge [29] could do the job as well. In such a scenario the monopole problem would be automatically solved, but we could not test it, since the $R$ charge would have been washed out at smaller temperatures, when the supersymmetry (and thus $R$ symmetry) breaking terms become important. This is why the existence of a large lepton number for solving the monopole problem is much more interesting. It is testable, at least in principle.

This mechanism of symmetry non-restoration could maybe also provide a way-out of the false vacuum problem of supersymmetric GUTs [30]. However, here the issue is somewhat subtle and must be addressed separately. The point is that it is not enough to keep the $\mathrm{SU}(5)$ unbroken above $T \approx M_{X}$;

one must also show that the vev of the adjoint $\mathbf{2 4}$ Higgs is non-zero and connects correctly to its physical value at small temperature.

We should also mention that symmetry non-restoration at high temper- 
ature may solve the domain wall problem of spontaneously broken discrete symmetries [31]. Now, in the MSSM this is not the case, but in many extensions of the theory one may have discrete symmetries. For example it can be spontaneous $\mathrm{CP}$ violation or a $Z_{3}$ [32] discrete symmetry of the next-tominimal supersymmetric standard model with the singlet field instead of a $\mu$ term in the superpotential. Clearly, the large lepton number is tailor-fit also for this task.

Another interesting question can be raised regarding the lepton number and neutrino masses. Namely, the solar and the atmospheric neutrino data point strongly towards nonvanishing neutrino masses [33]. If neutrino mass is of Dirac form, then of course it has no impact on the lepton number of the universe. If on the other hand neutrino mass is of Majorana nature, as many of us suspect and as suggested by the see-saw mechanism [34, then the situation becomes problematic. After all, Majorana masses break lepton number and one would expect any background lepton number to be washed out during the expansion of the universe. The issue however is more subtle and the outcome depends on the size of neutrino mass and the temperature range of one's interest [35.

\section{Acknowledgements}

We wish to acknowledge useful discussions with Charan Aulakh, Umberto Cotti and Alejandra Melfo. The work of B.B. was supported by the Ministry of Science and Technology of Slovenia and that of G.S. in part by EEC under the TMR contract ERBFMRX-CT960090. B.B. thanks ICTP for hospitality, where part of this work was done.

\section{References}

[1] H.-S. Kang and G. Steigman, Nucl. Phys. B372, 494 (1992), and references therein.

[2] J. Adams and S. Sarkar, talk presented at the ICTP Workshop on physics of relic neutrinos, Trieste, Italy, 1998, preprint OUTP-98-70P. 
[3] W.H. Kinney and A. Riotto, "Measuring the cosmological lepton asymmetry through the CMB anisotropy", hep-ph/9903459.

[4] J. Lesgourgues and S. Pastor, "Cosmological implications of a relic neutrino asymmetry", hep-ph/9904411.

[5] A.D. Linde, Phys. Rev. D14, 3345 (1976).

[6] B. Bajc, A. Riotto and G. Senjanović, Phys. Rev. Lett. 81, 1355 (1998), hep-ph/9710415.

[7] J. Liu and G. Segrè, Phys. Lett. B388, 259 (1994).

[8] I. Affleck and M. Dine, Nucl. Phys. B249, 361 (1985).

[9] A. Casas, W.Y. Cheng and G. Gelmini, Nucl. Phys. B538, 297 (1999), hep-ph/9709289.

[10] S. Weinberg, Phys. Rev., D9, 3357 (1974).

[11] R.N. Mohapatra and G. Senjanović, Phys. Rev. Lett. 42, 1651 (1979); Phys. Rev. D20, 3390 (1979); Phys. Lett. 89B, 57 (1979). For a recent review see for example G. Senjanović, COSMO 97, ed. L. Roszkowski, Singapore: World Scientific, 1998, p. 437, hep-ph/9805361.

[12] G. Dvali, A. Melfo and G. Senjanović, Phys. Rev. Lett. 75, 4559 (1995), hep-ph/9507230; P. Salomonson, B.S. Skagertan and A. Stern, Phys. Lett. B151, 243 (1985).

[13] P. Langacker and S.-Y. Pi, Phys. Rev. Lett. 45, 1 (1980).

[14] G. Bimonte and L. Lozano, Phys. Lett. B366, 248 (1996), hepth/9507079; Nucl. Phys. B460, 155 (1996), hep-th/9509060.

[15] G. Dvali and L. Krauss, "High Temperature Symmetry Breaking, SUSY Flat Directions, and the Monopole Problem", hep-ph/9811298.

[16] B. Bajc and G. Senjanović, "High Temperature Symmetry Breaking via Flat Directions", hep-ph/9811321.

[17] H. Haber, Phys. Rev. D46, 1317 (1982). 
[18] M. Mangano Phys. Lett. B147, 307 (1984).

[19] G. Dvali and K. Tamvakis, Phys. Lett. B378, 141(1996), hepph/9602336.

[20] B. Bajc, A. Melfo and G. Senjanović, Phys. Lett. B387, 796 (1996), hep-ph/9607242; B. Bajc and G. Senjanović, Nucl. Phys. Proc. Suppl. A52, 246 (1997), hep-ph/9610352.

[21] A. Riotto and G. Senjanović, Phys. Rev. Lett. 79, 349 (1997), hep$\mathrm{ph} / 9702319$.

[22] G. Gelmini and A. Kusenko, Phys. Rev. Lett. 82, 5202 (1999); hepph/9902354.

[23] H.E. Haber and H.A. Weldon, Phys. Rev. D25, 502 (1982).

[24] K.M. Benson, J. Bernstein and S. Dodelson, Phys. Rev. D44, 2480 (1991).

[25] L. Girardello, M.T. Grisaru and P. Salomonson Nucl. Phys. B178, 331 (1981).

[26] B.J. Harrington and A. Yildiz, Phys. Rev. Lett. 33, 324 (1974).

[27] B. Bajc, COSMO 97, ed. L. Roszkowski, Singapore: World Scientific, 1998, p. 467, hep-ph/9805352.

[28] Recently however, it has been claimed that even a small $L / S$ today (but large at $T>M_{W}$ ) may do the job, see J. McDonald, "Symmetry NonRestoration via Order $10^{-10} B$ and $L$ Asymmetries", hep-ph/9907358.

[29] B. Bajc, A. Riotto and G. Senjanović, Mod. Phys. Lett. A13, 2955 (1998), hep-ph/9803438.

[30] S. Weinberg, Phys. Rev. Lett. 48, 1776 (1982).

[31] G. Dvali and G. Senjanović, Phys. Rev. Lett. 74, 5178 (1995), hepph/9501387; G. Dvali, A. Melfo and G. Senjanović, Phys. Rev. D54, 7857 (1996), hep-ph/9601376. 
[32] See for example S.A. Abel, S. Sarkar and P.L. White, Nucl. Phys. B454, 663 (1995), hep-ph/9506359.

[33] For a review and references see e.g. A.Y. Smirnov, hep-ph/9901208.

[34] M. Gell-Mann, P. Ramond and R. Slansky in Supergravity (P. van Niewenhuizen and D. Freedman, eds.), (Amsterdam), North Holland, 1979; T. Yanagida in Workshop on Unified Theory and Baryon number in the Universe (O. Sawada and A. Sugamoto, eds.), (Japan), KEK, 1979; R.N. Mohapatra and G. Senjanović, Phys. Rev. Lett. 44, 912 (1980).

[35] P. Langacker, G. Segrè and S. Soni, Phys. Rev. D26, 3425 (1982); B. Bajc, U. Cotti and G. Senjanović, to appear. 